\title{
Instrumentalisations politiques et développementalistes du patrimoine culturel africain
}

Developmentalist and Political Instrumentalizations of African Cultural Heritage

Isabelle Brianso et Yves Girault

\section{(2) OpenEdition}

Journals

Édition électronique

URL : http://journals.openedition.org/edc/5766

DOI : $10.4000 /$ edc. 5766

ISSN : 2101-0366

Éditeur

Université de Lille

\section{Édition imprimée}

Date de publication : 1 juin 2014

Pagination : 149-162

ISBN : 978-2-917562-11-6

ISSN : $1270-6841$

Référence électronique

Isabelle Brianso et Yves Girault, «Instrumentalisations politiques et développementalistes du patrimoine culturel africain », Études de communication [En ligne], 42 | 2014, mis en ligne le 01 juin 2014, consulté le 10 décembre 2020. URL : http://journals.openedition.org/edc/5766 ; DOI : https:// doi.org/10.4000/edc.5766 


\section{ISABELLE BRIANSO ET YVES GIRAULT INSTRUMENTALISATIONS POLITIQUES ET DÉVELOPPEMENTALISTES DU PATRIMOINE CULTUREL AFRICAIN}

Les politiques coloniales en Afrique ont donné naissance à des espaces muséographiques dont les objectifs évoluaient d'une administration à l'autre. Parallèlement émergent des musées nationaux tournés, dès leur origine, vers les publics locaux ou encore des projets d'avant-garde où l'habitat traditionnel présente la diversité culturelle du territoire et des populations qui l'habitent.

Les constructions politiques et identitaires non linéaires qu'il serait ambitieux de prétendre résumer dans cet article sont marquées par des innovations (banque culturelle), mais aussi des déconstructions et des ruptures qui participent à l'élaboration d'une identité nationale au service du pouvoir en place comme moteur idéologique, puis de développement. La chute des Empires coloniaux va permettre à l'UNESCO de devenir progressivement un acteur de dialogue NordSud en matière culturelle. Ainsi, les vestiges de l'architecture coloniale font place à l'inscription de biens porteurs d'une identité locale sur la Liste du patrimoine mondial. Si les retombées économiques sont très largement attendues par les États, les populations locales cherchent encore leur place dans cette construction patrimoniale et cette mécanique politique. 


\section{Les premières structures muséales coloniales}

Les institutions muséales africaines sont indéniablement «associées à l'Occident et à son ancienne emprise coloniale» (Bouttiaux, 2007) et vont se multiplier à partir des années 1870. Selon Gaugue (1999a, 727) «à l'exception de l'Espagne en Guinée équatoriale, toutes les puissances européennes ont ouvert des musées dans leurs colonies d'Afrique tropicale, et lors des indépendances il existait une centaine de musées publics ». C'est ainsi que les Français ont créé à Saint-Louis (Sénégal) le premier musée d'Afrique tropicale en 1863 et au Bénin le premier musée en 1930. En Namibie, la plus ancienne structure muséale a été créée par les Allemands en 1907 à Windhoek, au Kenya l'«East Africa \& Uganda Natural History Society » a créé le « National Museum of Kenya » en 1910, au Nigéria, les premiers musées ont été créés par l'administration anglaise sous l'impulsion de Kenneth C. Murray, professeur d'art plastique.

Cependant, comme le souligne Gaugue (1999a, 727) « la fonction de ces musées et l'image de l'Afrique qu'ils diffusaient différaient d'une colonie à l'autre». Dans les colonies françaises, pétries des valeurs de modernité, les responsables de ces établissements avaient pour but de présenter et de conserver les témoins matériels des cultures des peuples dominés pour souligner, par contraste, la prétendue « supériorité » des civilisations européennes. Robert (2007) précise ainsi que le musée «tel qu'il fut conçu à l'époque précoloniale et coloniale renvoie à l'idée d'un 'primitivisme' sorte d'âge historique lointain et figé dans un temps d'avant le développement induit par le concept occidental de modernité ». À l'inverse, selon Gaugue (1999a, 727) «dans les colonies anglaises de Gold Coast (actuel Ghana) et du Nigeria, il fut un des lieux de valorisation du patrimoine africain destiné à favoriser l'émergence d'une conscience nationale et à préparer l'indépendance ». Si ces auteurs soulignent sans aucun doute la tendance générale de la finalité de ces structures muséales, certaines échappent à ce constat comme le musée national du Niger, inauguré le 18 décembre $1959^{1}$, qui avait pour principal objectif d'attirer et d'intéresser le public nigérien². Pablo Toucet (1963) précise que dans

\footnotetext{
$1 \quad$ Le Niger deviendra un État indépendant en 1960.

2 En 2008 en hommage à l'un de ses créateurs, ancien président de l'Assemblée nationale, il prendra le nom de Musée national Boubou-Hama.
} 
la ville de Niamey ont été regroupées, dans un espace important et au sein d'un musée de plein air, des reconstitutions de divers types d'habitats ruraux nigériens. Des parcelles de terrains ont été confiées à des familles issues de diverses populations pour qu'elles puissent construire leurs habitats traditionnels. Le visiteur pouvait ainsi déambuler au sein de campements nomades (Touaregs de l'Aït et de l'Azawak) de tentes Toubous, Peuls, de tentes communautaires et d'un habitat rural Haoussa (53\% de la population). Au bout de trois années d'activité, et selon Toucet (1963) ce musée aurait accueilli plus de 385000 visiteurs soit une moyenne journalière de 345 visiteurs.

Cette présentation bien trop lapidaire pourrait laisser croire qu'il n'existait aucune structure muséale réalisée par les communautés locales avant la colonisation, mais il n'en est rien. Dans une recherche antérieure (Girault et Galangau-Quérat, 2012) nous avions précisé que le Roi de Bamendjou (Cameroun) nous avait confié, qu'à l'époque, et dans les termes de sa langue locale, "peuh nôh » traduit en français par «le sac de la chefferie », désignait le lieu de stockage et de conservation du «patrimoine culturel» qui n'était cependant pas ouvert à tout un chacun.

Enfin, nous devons préciser qu'à cette époque les États coloniaux n'avaient pas l'exclusive des créations de musées. Comme le souligne Sabran $(1999,886-887)$ "à l'époque coloniale, créer ou soutenir la fondation d'un musée était le plus souvent, pour les détenteurs des pouvoirs traditionnels, une façon d'affirmer leur autorité face aux puissances coloniales». Elle précise ainsi qu'en 1922, «le Sultan Njoya créait à l'intérieur du Palais Royal de Foumban le premier musée camerounais pour [...] rappeler l'histoire du Royaume Bamun et affirmer, face à l'administration française, son autorité en tant que détenteur des pouvoirs traditionnels ».

\section{Une deuxième vague de créations-réhabilitations de musées au service de la construction d'États-nations}

Selon Anne Gaugue (1999b), il existait en 1999 un peu plus de 230 musées publics en Afrique tropicale dont $65 \%$ créés par des États indépendants et $35 \%$ par les autorités coloniales. Cet engouement des nouveaux États pour les musées n'était pas dénué d'intérêts politiques puisque l'objectif, le plus souvent reconnu, consistait à construire une identité nationale en gommant les spécificités 
historiques, linguistiques, ethnographiques, héritées de populations dont les territoires débordaient peи ou prou les nouvelles frontières imposées par les colonisateurs. Le musée participait alors à créer un « imaginaire collectif» (Anderson, 1996) pour faire émerger un sentiment d'appartenance à une communauté.

Cette instrumentalisation du musée au service d'un projet de nation s'observe, comme le souligne Gaugue (1999b), dans de nombreux pays africains. Elle étaye son point de vue en donnant quelques exemples :

A Lomé [Togo], le Musée national doit, selon le conservateur, « aider au raffermissement de la conscience nationale sans laquelle tout peuple demeure dans l'irresponsabilité et l'infantilisme » (Tchanilé, 1989: 4) [...]. Au Boswana où cette institution «contribue à sa modeste façon, à construire une nation» (Madondo, 1982: 190) et enfin en Zambie, elle est «un symbole de l'unité et de l'identité nationale » (Chellah, 1983 : 129) (Gaugue, 1999b, 337).

Il en est de même en Namibie où la proclamation de l'Indépendance le 20 mars 1990 a été accompagnée par l'inauguration d'une exposition permanente consacrée à l'Indépendance. Le musée rebaptisé en 1995 « National Museum of Namibia » abrite aujourd'hui une exposition sur l'histoire de la Namibie qui, selon Galaugau Quérat (et al., 2011), se conforme à l'idéologie nationale «Unity in Diversity ». Les symboles de la Nation (drapeau, constitution, hymne) sont, selon ces auteurs, présentés dans une muséographie austère, imposante et aussi dépouillée que l'espace de l'Indépendance est chargé. Gaugue (1999b) précise enfin que ces musées qui veulent bâtir une histoire nationale ont tendance à gommer tout ce qui pourrait contrecarrer l'objectif unitaire, les expositions privilégient donc le mythe de la non-violence et celui de la solidarité.

Ces diverses reproductions du modèle occidental du musée à des fins politiques ont mobilisé des intellectuels africains et des professionnels de musées et en 1991 Alpha Oumar Konaré, alors Président de l'ICOM, précisait "[qu'] il est grand temps, ce nous semble, de procéder à une totale remise en cause, il faut 'tuer', je dis tuer, le modèle occidental de musée en Afrique pour que s'épanouissent de nouveaux modes de conservation et de promotion du patrimoine » (Konaré, 1992). En communauté d'esprit, Tony Adedze (1992) défendait le fait que les musées africains devaient répondre à 
leurs aspirations et favoriser le processus de développement. Comme nous allons le préciser dans les lignes qui suivent, il nous semble qu'ils ont été entendus et c'est en 1999 à Lusaka (Zambie) que le Conseil international des musées africains (AFRICOM) est créé lors du colloque «Construire ensemble avec la communauté : un défi pour les musées africains ». Cette structure a pour «mission de contribuer au développement des sociétés africaines par la promotion du rôle des musées en tant que sources de culture et agents de la cohésion culturelle » (Nouvelles Africom, $n^{\circ}$ 1, 2002).

\section{Les musées au service du développement}

Ne pouvant présenter en quelques lignes un panel des « initiatives communautaires » (de Varine, 1991) réalisées à ce jour sur l'ensemble du continent africain, nous avons fait le choix de ne retenir que l'un des exemples les plus récents et spécifiques de l'Afrique, les banques culturelles.

L'objectif premier des banques culturelles développées à Fombori au Mali à partir de 1997 était de trouver une parade au fait que « pour pallier à leurs besoins immédiats, les populations rurales ont souvent recours au pillage des sites archéologiques et à la vente de leurs objets culturels aux collectionneurs d'objets d'art» (Daouda, 2007). Le principe de la banque culturelle est très simple : tout habitant qui dépose un objet dans le musée du village recevra, en contrepartie, un prêt d'argent afin d'entreprendre des activités génératrices de revenus. Les activités d'une banque culturelle s'articulent selon Daouda (2005) autour de trois éléments essentiels :

- Un musée villageois qui sert de lieu de collecte et de conservation. Les objets y sont authentifiés et documentés, puis inventoriés avant d'être présentés dans des expositions ;

- Une caisse de microcrédits. Cela donne l'opportunité aux populations d'utiliser leurs objets comme garantie pour l'obtention d'un prêt. La somme prêtée est fixée sur la base d'informations historiques et culturelles fournies par le propriétaire de l'objet. Le prêt est remboursé avec un faible taux d'intérêt. En absence de remboursement, l'objet devient la propriété de la communauté ;

- Un centre de formation culturelle qui met l'accent sur l'épanouissement socioculturel. Il propose des ateliers de formation en gestion, sur l'artisanat local, et donne des cours d'alphabétisation. 
Enfin, la banque culturelle est une institution communautaire gérée par une association villageoise. À ce jour, il existe quatre banques culturelles au Mali (Fombori, Kola, Dégnékoro et Dimbal) et deux nouveaux projets ont vu le jour à Koutammakou (Togo, 2011) et Taneka (Bénin, 2012).

Ces projets muséologiques africains se mettent en place dans un but de développement local à l'instar des politiques de valorisation de biens culturels et naturels inscrits sur la Liste du patrimoine mondial (UNESCO, 1972). Avec la chute progressive des empires coloniaux, l'UNESCO, comme nous allons le préciser, va occuper peu à peu un rôle diplomatique Nord-Sud de premier plan comme dynamique de coopération autour de grands chantiers de développement basés sur la sauvegarde, la transmission et la valorisation des patrimoines pour les générations futures. Néanmoins, ces politiques développementalistes suscitent en même temps des déséquilibres locaux (spéculations foncières, supra-reconnaissance du statut de l'« expert » au détriment des savoirs autochtones, prédominance de la matérialité des objets du patrimoine et marginalisation des communautés locales) instrumentalisés par les pays africains et occidentaux.

\section{La création d'une « Société des esprits »: le « patrimoine » au cour d'une fabrique de développement tout au long du $\mathrm{XX}^{\mathrm{e}}$ siècle}

\section{Brève genèse d'une coopération intellectuelle}

L'Organisation des Nations Unies pour l'éducation, la science et la culture mieux connue sous l'acronyme de «UNESCO » est le fruit d'une construction intellectuelle et diplomatique complexe au cours des grandes dates de l'histoire des relations internationales de la première moitié du $\mathrm{XX}^{\mathrm{e}}$ siècle. La période de l'entre-deuxguerres, marquée par une stabilité mondiale relative, servira de levier à une nouvelle impulsion intellectuelle sous l'égide de la Société des Nations (SDN), à l'heure où les Empires coloniaux connaissent leurs premières fissures face à une Europe affaiblie qui conduiront, quelques décennies plus tard, à la décolonisation. La France et le Royaume-Uni, rivaux diplomatiques de la première heure pour asseoir leur hégémonie impériale, vont continuer à marquer 
leurs différences stratégiques dans la construction d'une coopération intellectuelle défendue en premier lieu par la France et soutenue par le Conseil et l'Assemblée de la Société des Nations, afin d'étendre le rôle de la SDN et d'œuvrer en faveur de la paix. Elle disparaitra en 1946 pour laisser place à l'UNESCO en tant qu'organisation intergouvernementale rattachée aux Nations Unies, continuité légitime de la SDN sous la pression de l'Angleterre et des États-Unis devenus la nouvelle puissance mondiale.

Les années de guerre rendent légitime une «solidarité intellectuelle et morale de l'humanité » pour construire la paix. L'Acte constitutif de l'UNESCO (1946) sera le socle sur lequel reposent les valeurs de justice, de libertés fondamentales, de paix et de sécurité pour les peuples. Si le caractère apolitique de l'UNESCO est ainsi dressé au nom des nations entières dans ces trois domaines d'action, l'organisation va cependant être confrontée rapidement à des enjeux politiques internationaux de première importance dans un monde changeant et instable, au sein duquel les grandes puissances s'affrontent. Les programmes de paix et de dialogue entre les cultures masquent péniblement des tensions diplomatiques internes propres à l'organisation, rythmées par une vision du monde d'abord ethnocentrée, puis ébranlée par la montée du communisme, du conflit Est-Ouest, de la décolonisation et de l'affirmation progressive du Tiers-Monde comme nouvel acteur stratégique sur une scène internationale (Maurel, 2006).

L'organisation s'enlise dans une diplomatie tendue entre les blocs géopolitiques, loin de ses préoccupations universalistes initiales. Le succès de la Campagne de Nubie (1960-1980) donne un nouveau souffle à l'UNESCO, en tant que coordinateur des grands travaux de sauvetage et intermédiaire diplomatique entre deux de ses États parties (Égypte, Soudan) et le reste de la communauté internationale. Le socle d'un patrimoine culturel «commun à l'humanité » qu'il faut sauvegarder puis transmettre aux générations futures est posé. La Convention concernant la protection du patrimoine mondial, culturel et naturel (UNESCO, 1972), bien que mariant deux biens d'un genre nouveau (nature et culture), s'appuie sur ce contexte international favorable et dote les États membres de l'organisation d'une responsabilité politique de mise en œuvre des normes comme relais local au détriment des populations. Dans un premier temps, les traces matérielles de la colonisation vont servir de leviers d'inscription aux États africains (Sénégal, Mozambique, etc.) avant 
de revendiquer des sites plus caractéristiques de leur patrimoine local (Mali, Mauritanie, Namibie, Togo, etc.). Une instrumentalisation partagée dans un contexte mondialisé où seules les retombées socio-économiques et touristiques sont attendues par les pays. Une nouvelle alliance «État, Bailleurs, Population» naît sur ces sites labélisés, impulsant des stratégies de coopération comme moteur de développement avec comme unique interlocuteur, les représentants des États concernés, ce qui dans les nouveaux États-nations renforce leur légitimité face aux populations locales en quête identitaire.

\section{Le Label «patrimoine mondial de l'humanité » : ébauche d'un dialogue diplomatique Nord-Sud et d'une instrumentalisation partagée}

La ratification, la mise en œuvre et l'essor de la Convention concernant la protection du patrimoine mondial, culturel et naturel (UNESCO, 1972) vont donner à l'UNESCO un rôle de dialogue Nord-Sud de premier plan. Ce texte est un instrument juridique qui pose et définit le cadre légal international de la notion de « patrimoine mondial » devant être transmis aux générations futures. Il permet de proposer puis d'inscrire sur la Liste du patrimoine mondial des biens culturels et naturels ayant une "valeur universelle et exceptionnelle $»^{3}$. Cette Liste, non exhaustive, doit encourager les États à préserver, sauvegarder, mettre en valeur et transmettre les patrimoines locaux aux générations futures qui font le lien entre passé, présent et futur (Brianso, 2012).

En 2013, la Liste du patrimoine mondial compte 981 biens inscrits, dont 759 biens culturels, 193 biens naturels et 29 sites mixtes, répartis dans 160 États parties ayant ratifié ce texte normatif. Le découpage géographique des biens inscrits sur la Liste est organisé par « région», elles sont au nombre de cinq (Afrique ; États arabes ;

3 La valeur universelle exceptionnelle signifie une importance culturelle et/ ou naturelle tellement exceptionnelle qu'elle transcende les frontières nationales et qu'elle présente le même caractère inestimable pour les générations actuelles et futures de l'ensemble de l'humanité. À ce titre, la protection permanente de ce patrimoine est de la plus haute importance pour la communauté internationale tout entière. Le Comité définit les critères pour l'inscription des biens sur la Liste du patrimoine mondial. (Orientations, 2012, 49). 
Asie et Pacifique ; Europe et Amérique du Nord ; Amérique latine et Caraïbes).

La région «Afrique » comptabilise un faible nombre d'inscriptions avec 86 sites inscrits en 2012, dont 47 biens culturels, 35 biens naturels et 4 sites mixtes répartis dans 32 États parties. La progression annuelle du nombre de biens inscrits sur la Liste est constante pour cette région: d'un à sept sites inscrits chaque année entre 1978 et 2012. Ces données indiquent la sous-représentation du patrimoine culturel africain en comparaison avec les autres aires géographiques. Un certain nombre d'États africains n'ont encore jamais inscrit de bien (culturel, naturel ou mixte) depuis l'adoption et la ratification de la Convention, comme l'Angola, le Burundi, la Guinée équatoriale ou encore le Libéria. Les causes peuvent être plurielles, comme l'instabilité politique, les conflits armés ou encore un non-savoirfaire institutionnel et technique répondant aux normes onusiennes: création d'un inventaire national, expertise pour la soumission d'un formulaire, etc. (ICOMOS, 2000, 19).

À l'inverse, d'autres États africains ont rapidement développé une politique d'inscriptions à l'instar du Sénégal. En effet, ce pays a constitué une Liste indicative composée de huit ${ }^{4}$ biens pouvant être présentés lors du Comité du patrimoine mondial. En 2013, le Sénégal compte sept biens inscrits sur la Liste du patrimoine mondial, dont cinq biens culturels, un bien naturel et un bien mixte. Le premier site inscrit fut l'île de Gorée (1978) en tant que bien « offrant un exemple éminent d'un type de construction ou d'ensemble architectural ou technologique ou de paysage illustrant une ou des périodes significatives de l'histoire humaine » (UNESCO, Orientations 2012, critère (iv)). Cette lecture matérielle du patrimoine va rencontrer certaines réticences auprès des «experts » du patrimoine africain lors de réunions de travail au sein de l'UNESCO. Néanmoins, à la fin des années 70, l'inscription de l'île de Gorée par l'UNESCO est un message culturel et diplomatique pour la reconnaissance de l'un des centres de commerce d'esclaves à des fins de dialogue de réconciliation Nord-Sud.

4 Les huit biens inscrits sur la Liste indicative (révisée en 2005) : l'Aéropostale, l'île de Carabane, Architecture rurale de Basses-Casamance : les cases à impluvium du royaume bandial, Parc national des îles de La Madeleine, les Escales du fleuve Sénégal, les tumulus de Cekeen, le Lac Rose, le Vieux Rufisque. 
En 2004, alors que l'UNESCO célébrait l'« Année internationale de commémoration de la lutte contre l'esclavage et de son abolition » un itinéraire culturel et route d'échange avait déjà été lancé dix ans plus tôt sur ce même thème : la Route des esclaves. En effet, est adopté en 1994 à Madrid le concept de « route » ou d' "itinéraire » en tant qu' "éléments matériels qui doivent leur valeur culturelle aux échanges et à un dialogue multidimensionnel entre les pays ou régions, et qui illustrent l'interaction du mouvement, tout au long de la route, dans l'espace et le temps » (UNESCO, Orientations 2012, 23). La Route des esclaves comprend un itinéraire géographique, une histoire humaine, coloniale et commerciale, séculaire, mais aussi une dimension immatérielle. De même, la ville de Saint-Louis du Sénégal reflète l'architecture coloniale comme modèle urbain français en Afrique (Sow, 2011). Elle est inscrite sur la Liste du patrimoine mondial en 2000 selon deux critères, notamment en tant « [qu'] ancienne capitale de l'Afrique occidentale, est un remarquable exemple de ville coloniale, caractérisé par un cadre naturel particulier, et illustre le développement du gouvernement colonial dans la région » (ICOMOS, 2000, 47). Menacée par son état de conservation général, la ville s'inscrit néanmoins dans une dynamique locale de développement en phase avec une Afrique contemporaine en mutation.

En effet,

[...] la spéculation foncière en cours et le développement du tourisme à Saint-Louis révèlent que le classement de l'île sur la liste du patrimoine mondial de l'UNESCO a des effets de levier [...]. S'il y a eu « régression » et « déclin », comme se complaisent à le clamer les partisans de la ville-musée, peut-être est-ce dû à l'effacement des seuls aspects européens que revêtait Saint-Louis ? N'en déplaise à certains «conservateurs de musée » reconvertis sous les tropiques en «urbaniste spécialiste du patrimoine colonial», les analyses révèlent que la ville européenne s'estompe depuis les premières années de l'Indépendance pour faire place progressivement à un autre modèle de ville sénégalais, fait de symbiose, d'adaptation, de complémentarité, de créativité et qui pourrait bien être celui de l'avenir dans une Afrique en pleine mutation [...] (Sow, 2011, 103104). 


\section{Conclusion}

L'évolution et l'extension du concept de «patrimoine » grâce aux échanges, réunions et discussions entre «experts» à la lecture de rapports de suivi et d'évaluation en provenance de la société civile, ont permis aux institutions internationales (UNESCO, ICOMOS, ICOM, AFRICOM) de s'interroger sur les nouvelles définitions du terme «patrimoine » et les processus de patrimonialisation à partir de bonnes pratiques de gestion dictées par les normes. Ce mécanisme endogène a cependant été confronté à un questionnement bottomup majeur: la participation active des «communautés » dans ce nouveau paysage culturel africain. Notons que les recommandations relatives à l'implication communautaire en tant que partage de savoirs Nord-Sud sont désormais de plus en plus mises en avant par les grandes instances internationales en tant qu'enjeux éthique, sociétal et patrimonial. L'UNESCO célébrait en 2012 le $40^{\mathrm{e}}$ anniversaire de la Convention de 1972 sur le thème : «Le patrimoine mondial et le développement durable : le rôle des communautés locales ».

Ainsi, les pratiques patrimoniales participatives, bien que critiquées par certains chercheurs (Perkin, 2010; Watson, 2007), semblent se présenter comme un compromis interculturel acceptable et évolutif entre des modèles normatifs occidentaux rigides et des populations locales trop longtemps marginalisées. En effet, seule la remise en question des standards occidentaux et des pratiques des professionnels en charge de leur mise en œuvre pourrait conduire à une éthique patrimoniale basée sur l'hybridation (savoirs, expertise) qui, selon Paquette (2012) serait «susceptible et garante de produire un rapport patrimonial interculturel véritablement inclusif ».

Du discours de Alpha Oumar Konaré en 1992 « [...], il faut 'tuer', je dis tuer, le modèle occidental de musée en Afrique [...]» aux innovations locales (banque culturelle), les États africains impulsent et instrumentalisent des dynamiques communautaires comme nouvelle forme locale de conservation durable du patrimoine en faveur de politiques développementalistes.

ISABELLE BRIANSO

Université Autonome de Barcelone (UAB, Espagne) UMR 208 Patrimoines locaux (PALOC) MNHN/IRD

YVES GIRAULT

Muséum national d'Histoire naturelle (MNHN) UMR 208 Patrimoines locaux (PALOC) MNHN/IRD 


\section{BIBLIOGRAPHIE}

Anderson B. R., (1996), L'imaginaire national : réflexion sur l'origine et l'essor du nationalisme, Paris, La découverte, 224 p.

Adeze A. T., (1992), Collection et définition du patrimoine culturel au Togo ?, in Actes du colloque Quels musées pour l'Afrique, patrimoine en devenir ?, Édition ICOM, pp. 173-176.

Bocco J., (1999), La route des esclaves, in La Convention du patrimoine mondial et les paysages culturels en Afrique. Actes de la réunion d'experts, 9-14 mars, Kenya, UNESCO, pp. 74-78.

Bouttiaux A. M., (2007), Les musées en Afrique, nouvelles plate-formes d'accès à la culture, in Bouttiaux A. M. (éd.), Afrique : musées et patrimoines pour quels publics ?, pp. 9-10.

Bortolotto C. (sous la dir.), (2011), Le patrimoine culturel immatériel. Enjeux d'une nouvelle catégorie, éditions Maison des sciences de l'homme, Paris, 252 p.

Brianso I., (2012), La médiation culturelle appliquée aux sites du patrimoine mondial. Vers une pédagogie interculturelle, in Éditions La Découverte Les nouveaux enjeux des politiques culturelles. Dynamiques européennes, pp. 269-283.

Brianso I., (2010), Les politiques internationales de valorisation pour des biens culturels inscrits sur la Liste du patrimoine mondial de l'UNESCO. Étude de cas à Angkor (Cambodge) : médiation culturelle, coopération et interculturalité, Thèse de doctorat (Label européen) en sciences de l'information et de la communication, Université de Versailles Saint-Quentin-en-Yvelines, 492 p.

Chaumier S., (2005), L'identité, un concept embarrassant constitutif de l'idée de musée, Cultures \& musées $\mathrm{n}^{\circ}$ 6, pp. 21-42.

Chellah M., (1983), Le musée national de Zambie à Living Stone, Museum, n² 2, $129 \mathrm{p}$.

De Varine H., (1991), L'initiative communautaire, Paris, Éditions W/MNES, 265 p.

Galangau-Quérat F., Nivart A. et al., (2011), Les musées en Namibie au cœur d'une société en mutation, in Rasse A. et Girault Y. (Coord.), Les musées aux prismes de la communication, Hermès $n^{\circ} 6$, CNRS Éditions, pp. 148-153.

Gaugue A., (1999a), Musées et colonisation en Afrique tropicale, Cahier d'études africaines, Vol. 39, Cahier 155/156 Prélever, exhiber, la mise en musées, pp. 727-745. Gaugue A., (1999b), La mise en scène de la nation dans les musées d'Afrique tropicales, in Ethnologie française, nouvelle série, T. $29^{\mathrm{e}}, \mathrm{n}^{\circ} 3^{\mathrm{e}}$, Musée Nation : après les colonies, Presses Universitaires de France, pp. 337-344.

Girault Y. et Galangau-Quérat F., (2012), Problématiques et enjeux de l'expographie du patrimoine culturel et proto-industriel dans les musées de la route des chefferies au Cameroun, in Processus, problématiques, enjeux du patrimoine industriel, Laura Teodora Ghinea (éd.), Ed. Eikon, pp. 164-180.

Girault Y. et Galangau-Quérat F., (2011), Identités culturelles et Patrimoines : Perspectives muséologiques, in Rodriguez Barbosa C., Alvarenga L. et Abrantes Baracho R. M. (éds.), II Seminario Internacional Ciencia e Museologia: Universo Imaginario, Universidade Federal do Minas Geraïs, Brasil, pp. 7-21.

ICOMOS, (2000), La Convention du patrimoine et les paysages culturels en Afrique, in Réunion d'experts, 9-14 mars 1999, Tiwi, Kenya, 106 p. 
ICOMOS, (2012), Le patrimoine, moteur de développement, in Actes du symposium de la XVII assemblée générale de l'ICOMOS, 27 novembre-2 décembre, Paris, UNESCO, $1416 \mathrm{p}$.

Keita D., (2005), Guide de la Banque Culturelle basé sur les expériences au Mali, 37 p. : www.epa-prema.net/documents/.../guide_banque_culturelle.pdf (consulté en août 2013).

Keita D., (2007), Les banques culturelles : une nouvelle initiative de protection du patrimoine culturel et de développement des localités, in Bouttiaux A. M. (éd.), Afrique : musées et patrimoines pour quels publics ?, pp. 117-127.

Konaré A. O., (1992), Allocution, in Actes du colloque Quels musées pour l'Afrique, patrimoine en devenir, Édition ICOM, pp. 385-387.

Madondo T. W., (1982), Au Botswana, le musée à la rencontre de la population, Museum, $3: 190 \mathrm{p}$.

Maurel C., (2006), L'UNESCO de 1945 à 1974, Thèse de doctorat en histoire contemporaine, Université Paris 1, Paris, 1493 p.

Mauss M., (1969), La nation, Euvres, Vol. 3, Paris, Éditions de minuit, 738 p.

Morin E., (1990), Introduction à la pensée complexe, Paris, Ed. Du Seuil, 158 p.

Paquette J., (2012), «Expertise et patrimoine autochtone : hybridation des savoirs et évolutions récentes des pratiques patrimoniales en Nouvelle-Zélande », Éthique publique [En ligne], vol. 14, $\mathrm{n}^{\circ} 1-2012$, mis en ligne le 03 février 2013, consulté le 06 juin 2013. URL : http://ethiquepublique.revues.org/974 ; DOI : 10.4000/ ethiquepublique.974.

Pemberton J.-A., (2007), Vers une Société des esprits : de la Commission internationale de coopération intellectuelle à l'UNESCO, in 60 ans d'histoire de l'UNESCO ; Actes du colloque international, 16-18 novembre 2005, Paris, UNESCO, pp. 67-75.

Perkin C., (2010), Beyond the rhetoric: negotiating the politics and realising the potential of community-driven heritage engagement, International Journal of Heritage Studies, Vol. 16, n 1-2, pp. 107-122.

Renoliet J.-J., (2007), L'UNESCO oubliée : l'Organisation de coopération intellectuelle (1921-1946), in 60 ans d'histoire de 1'UNESCO; Actes du colloque international, 16-18 novembre 2005, Paris, UNESCO, pp. 61-66.

Robert Y., (2007), Musées en Afrique entre universalité et singularité : réflexions et propositions, in Bouttiaux A. M. (éd.), Afrique : musées et patrimoines pour quels publics ?, pp. 13-33.

Sabran M., (1999), La maison du pays : l'exposition du patrimoine dans les musées privés d'Afrique de l'Ouest et du Cameroun. Cahier d'études africaines, Vol. 39, 155/156 Prélever, exhiber, la mise en musées, pp. 885-903.

Sow A., (2011), Saint-Louis du Sénégal : L'île ou la ville?, in Le patrimoine, moteur de développement, Actes du symposium de la XVII ${ }^{\mathrm{e}}$ assemblée générale de l'ICOMOS, 27 novembre-2 décembre, Paris, UNESCO, pp. 98-105.

Tchanilé M., (1989), Musée et développement culturel, Actes du séminaire-atelier de Kpaline, Patrimoine muséal : facteur de développement endogène, Lomé. Direction du Musée national des sites et des monuments.

Toucet P., (1963), Le Musée national de la République du Niger, Niamey, Museum, 16 (3), pp. 192-196. 
UNESCO, (2000), Rapport du Comité du patrimoine mondial, in Actes de la vingtquatrième session, 27 novembre-2 décembre, Cairns, Australie, 145 p.

UNESCO, (2007), Patrimoine mondial. Défis pour le millénaire, Paris, UNESCO, $200 \mathrm{p}$.

UNESCO, (2012), Orientations devant guider la mise en æuvre de la Convention du patrimoine mondial, Paris, UNESCO, $168 \mathrm{p}$.

Watson S., (2007), Museums and their Communities, Londres, Routledge, 592 p. 